\title{
AN EXTENSION OF SUB-FRACTIONAL BROWNIAN MOTION
}

\author{
Aissa SGHIR
}

\begin{abstract}
In this paper, firstly, we introduce and study a self-similar Gaussian process with parameters $H \in(0,1)$ and $K \in(0,1]$ that is an extension of the well known sub-fractional Brownian motion introduced by Bojdecki et al. [4]. Secondly, by using a decomposition in law of this process, we prove the existence and the joint continuity of its local time.
\end{abstract}

2010 Mathematics Subject Classification: 60G18.

Key words: Sub-fractional Brownian motion, bifractional Brownian motion, fractional Brownian motion, local time, local nondeterminism.

\section{Introduction}

The sub-fractional Brownian motion $S^{H}:=\left\{S_{t}^{H} ; t \geq 0\right\}$ ( $\mathrm{sfBm}$ for short) with parameter $H \in(0,2)$ was introduced by Bojdecki et al. [4]. It is a continuous centered Gaussian process, starting from zero, with covariance function

$$
\mathbb{E}\left(S_{t}^{H} S_{s}^{H}\right)=t^{H}+s^{H}-\frac{1}{2}\left[(t+s)^{H}+|t-s|^{H}\right] .
$$

The case $H=1$ corresponds to the standard Brownian motion (Bm for short).

Bojdecki et al. [4] have proved that the increments of sfBm satisfy

$$
C_{H}^{\prime}|t-s|^{H} \leq \mathbb{E}\left[S_{t}^{H}-S_{s}^{H}\right]^{2} \leq C_{H}|t-s|^{H} .
$$

On the other hand, Ruiz de Chávez and Tudor [8] have obtained for $H \in(0,1)$ the following decomposition in law of sfBm

$$
S_{t}^{H} \stackrel{d}{=} B_{t}^{H}+C_{1}(H) X_{t}^{H},
$$

where $C_{1}(H)=\sqrt{\frac{H}{2 \Gamma(1-H)}}$ and $X_{t}^{H}=\int_{0}^{+\infty}\left(1-e^{-\theta t}\right) \theta^{-\frac{H+1}{2}} d W_{\theta}$, and the $\mathrm{Bm} W$ and the fractional Brownian motion (fBm for short) $B^{H}$ are independent. 
The case $H \in(1,2)$ is given by Bardina and Bascompte [1]. They proved that

$$
B_{t}^{H} \stackrel{d}{=} S_{t}^{H}+C_{2}(H) X_{t}^{H},
$$

where $C_{2}(H)=\sqrt{\frac{H(H-1)}{2 \Gamma(2-H)}}$, and the $\mathrm{Bm} W$ and the $\operatorname{sfBm} S^{H}$ are independent.

They also proved that the process $X^{H}$ is Gaussian, centered, and that its covariance function is

$$
\mathbb{E}\left(X_{t}^{H} X_{s}^{H}\right)= \begin{cases}\frac{\Gamma(1-H)}{H}\left[t^{H}+s^{H}-(t+s)^{H}\right], & \forall H \in(0,1), \\ \frac{\Gamma(2-H)}{H(H-1)}\left[(t+s)^{H}-t^{H}-s^{H}\right], & \forall H \in(1,2) .\end{cases}
$$

Moreover, Mendy [7] proved that there exists a constant $C_{K}>0$ such that

$$
\mathbb{E}\left[X_{t}^{K}-X_{s}^{K}\right]^{2} \leq C_{K}|t-s|^{2} .
$$

The self similarity and stationarity of the increments are two main properties for which $\mathrm{fBm}$ enjoyed success as modeling tool in telecommunications and finance. The $\mathrm{sfBm}$ is an extension of $\mathrm{Bm}$ which preserves many properties of $\mathrm{fBm}$, but not the stationarity of the increments. This property makes sfBm a possible candidate for models which involve long dependence, self similarity and non stationarity of increments. It is, thus, very natural to explore the existence of processes which keep some of the properties of sfBm, specially a decomposition in law that includes $\mathrm{sfBm}$, but also enlarge our modelling tool kit. The same motivation is given by Houdré and Villa [6] in case of the bifractional Brownian motion (bfBm for short), which generalizes the $\mathrm{fBm}$.

Definition 1.1. We denote by $S^{H, K}:=\left\{S_{t}^{H, K} ; t \geq 0\right\}$ a centered Gaussian process, starting from zero, with covariance function

$$
S(t, s):=\mathbb{E}\left(S_{t}^{H, K} S_{s}^{H, K}\right)=\left(t^{H}+s^{H}\right)^{K}-\frac{1}{2}\left[(t+s)^{H K}+|t-s|^{H K}\right],
$$

where $H \in(0,1)$ and $K \in(0,1]$.

The case $K=1$ corresponds to sfBm with parameter $H \in(0,1)$.

Existence of $S^{H, K}$ can be shown in the following two ways: 1) Consider the process

$$
Y_{t}:=\frac{B_{t}^{H, K}+B_{-t}^{H, K}}{2^{2-K}}, \quad t \geq 0
$$

where $\left\{B_{t}^{H, K} ; t \in \mathbb{R}\right\}$ is the bfBm on the whole real line with parameters $H \in(0,1)$ and $K \in(0,1]$, introduced by Houdré and Villa [6]. It is easy to see that $Y_{t}$ and $S^{H, K}$ have the same covariance function. Therefore 
$S^{H, K}$ exists. 2) It can be shown by the following theorem which gives also a decomposition in law of the process. It will be useful in the sequel.

Theorem 1.2. 1) For any $H \in(0,1)$ and $K \in(0,1]$, the function $S(.,$. is symmetric and positive definite.

2) We have the following decomposition in law

$$
S_{t}^{H K} \stackrel{d}{=} S_{t}^{H, K}+C_{3}(K) X_{t^{H}}^{K},
$$

where $C_{3}(K)=\sqrt{\frac{K}{\Gamma(1-K)}}, H \in(0,1)$, and $K \in(0,1)$, and $S^{H, K}$ and the $B m W$ are independent. $S^{H K}$ is sfBm with parameter $H K \in(0,1)$.

Proof: 1) The case $K=1$ corresponds to sfBm with parameter $H \in(0,1)$.

First recall the following (easily verified) identity

$$
\lambda^{K}=\frac{K}{\Gamma(1-K)} \int_{0}^{+\infty}\left(1-e^{-\lambda x}\right) x^{-1-K} d x,
$$

valid for $\lambda \geq 0$ and $K \in(0,1)$. Then, for any $c_{1}, c_{2}, \ldots, c_{n} \in \mathbb{R}$,

$$
\begin{aligned}
\sum_{i=1}^{n} \sum_{j=1}^{n} c_{i} c_{j} S\left(t_{i}, t_{j}\right) \\
=\frac{K}{\Gamma(1-K)} \int_{0}^{+\infty} \sum_{i=1}^{n} \sum_{j=1}^{n} c_{i} c_{j}\left(-e^{-x\left(t_{i}^{H}+t_{j}^{H}\right)}+\frac{1}{2} e^{-x\left(t_{i}+t_{j}\right)^{H}}+\frac{1}{2} e^{-x\left|t_{i}-t_{j}\right|^{H}}\right) x^{-1-K} d x \\
=\frac{K}{2 \Gamma(1-K)} \int_{0}^{+\infty} \sum_{i=1}^{n} \sum_{j=1}^{n} c_{i} c_{j} e^{-x\left(t_{i}^{H}+t_{j}^{H}\right)}\left(e^{x\left(t_{i}^{H}+t_{j}^{H}-\left(t_{i}+t_{j}\right)^{H}\right)}-1\right) x^{-1-K} d x \\
\quad+\frac{K}{2 \Gamma(1-K)} \int_{0}^{+\infty} \sum_{i=1}^{n} \sum_{j=1}^{n} c_{i} c_{j} e^{-x\left(t_{i}^{H}+t_{j}^{H}\right)}\left(e^{x\left(t_{i}^{H}+t_{j}^{H}-\left|t_{i}-t_{j}\right|^{H}\right)}-1\right) x^{-1-K} d x .
\end{aligned}
$$

Since the functions $t^{H}+s^{H}-(t+s)^{H}$ and $t^{H}+s^{H}-|t-s|^{H}$ are positive definite, so are

$$
\left(e^{x\left(t^{H}+s^{H}-(t+s)^{H}\right)}-1\right) \text { and }\left(e^{x\left(t^{H}+s^{H}-|t-s|^{H}\right)}-1\right),
$$

for all $x \geq 0$. Therefore the function $S(.,$.$) is positive definite.$

2) Using the fact that $S^{H, K}$ is a Gaussian process, it suffices to see that

$$
\mathbb{E}\left[S_{t}^{H K} S_{s}^{H K}\right]=\left(t^{H K}+s^{H K}-\left(t^{H}+s^{H}\right)^{K}\right)+\mathbb{E}\left[S_{t}^{H, K} S_{s}^{H, K}\right] .
$$

In the sequel $C$ and $C_{p}$ denote constants which will be different even when they vary from one line to the next. 


\section{Local time of $S^{H, K}$}

We begin this section by the definition of local time. For a complete survey on local time, we refer to Geman and Horowitz [5] and the references therein.

Let $X:=\left\{X_{t} ; t \geq 0\right\}$ be a real-valued separable random process with Borel sample functions. For any Borel set $B \subset \mathbb{R}^{+}$, the occupation measure of $X$ on $B$ is defined as

$$
\mu_{B}(A)=\lambda\left\{s \in B ; X_{s} \in A\right\}, \quad \forall A \in \mathcal{B}(\mathbb{R}),
$$

where $\lambda$ is the one-dimensional Lebesgue measure on $\mathbb{R}^{+}$. If $\mu_{B}$ is absolutely continuous with respect to Lebesgue measure on $\mathbb{R}$, we say that $X$ has a local time on $B$ and define its local time, $L(B,$.$) , to be the$ Radon-Nikodym derivative of $\mu_{B}$. Here, $x$ is the so-called space variable and $B$ is the time variable. By standard monotone class arguments, one can deduce that the local time has a measurable modification that satisfies the occupation density formula: for every Borel set $B \subset \mathbb{R}^{+}$and every measurable function $f: \mathbb{R} \rightarrow \mathbb{R}^{+}$,

$$
\int_{B} f\left(X_{t}\right) d t=\int_{\mathbb{R}} f(x) L(B, x) d x .
$$

Sometimes, we write $L(t, x)$ instead of $L([0, t], x)$.

Here is the outline of the analytic method used by Berman $[\mathbf{2}]$ for the calculation of the moments of local time.

For fixed sample function at fixed $t$, the Fourier transform on $x$ of $L(t, x)$ is the function

$$
F(u)=\int_{\mathbb{R}} e^{i u x} L(t, x) d x .
$$

Using the density of occupation formula, we get

$$
F(u)=\int_{0}^{t} e^{i u X_{s}} d s .
$$

Therefore, we may represent the local time as the inverse Fourier transform of this function, i.e.,

$$
L(t, x)=\frac{1}{2 \pi} \int_{-\infty}^{+\infty}\left(\int_{0}^{t} e^{i u\left(X_{s}-x\right)} d s\right) d u .
$$

We end this section by the definition of the concept of local nondeterminism, (LND for short). Let $J$ be an open interval on the $t$ axis. Assume that $\left\{X_{t} ; t \geq 0\right\}$ is a zero mean Gaussian process without singularities 
in any interval of the length $\delta$, for some $\delta>0$, and without fixed zeros, i.e., there exists $\delta>0$, such that

$$
\begin{cases}\mathbb{E}\left[X_{t}-X_{s}\right]^{2}>0, & \text { whenever } 0<|t-s|<\delta, \\ \mathbb{E}\left(X_{t}\right)^{2}>0, & \text { for } t \in J .\end{cases}
$$

To introduce the concept of LND, Berman [3] defined the relative conditioning error,

$$
V_{p}=\frac{\operatorname{Var}\left\{X_{t_{p}}-X_{t_{p-1}} / X_{t_{1}}, \ldots, X_{t_{p-1}}\right\}}{\operatorname{Var}\left\{X_{t_{p}}-X_{t_{p-1}}\right\}},
$$

where for $p \geq 2, t_{1}<\cdots<t_{p}$ are arbitrary ordered points in $J$.

We say that the process $X$ is LND on $J$ if for every $p \geq 2$,

$$
\lim _{c \rightarrow 0^{+}} \inf _{0<t_{p}-t_{1} \leq c} V_{p}>0 .
$$

This condition means that a small increment of the process is not almost relatively predictable on the basis of a finite number of observations from the immediate past. Berman [3] has proved, for Gaussian processes, that the LND is characterized as follows.

Proposition 2.1. A Gaussian process $X$ is $L N D$ if and only if for every integer $p \geq 2$, there exist two positive constants $\delta$ and $C_{p}$ such that

$$
\operatorname{Var}\left(\sum_{i=1}^{p} u_{j}\left(X_{t_{j}}-X_{t_{j-1}}\right)\right) \geq C_{p} \sum_{i=1}^{m} u_{j}^{2} \operatorname{Var}\left(X_{t_{j}}-X_{t_{j-1}}\right),
$$

for all orderer points $t_{1}<\cdots<t_{p}$ that are arbitrary points in $J$ with $t_{0}=0, t_{p}-t_{1} \leq \delta$ and $\left(u_{1}, \ldots, u_{j}\right) \in \mathbb{R}$.

Remark 2.2. Mendy [7] proved by using (1) that the sfBm is LND on $[0,1]$ for any $H \in(0,1)$.

The purpose of this section is to present sufficient conditions for the existence of the local time of $S^{H, K}$. Furthermore, using the LND approach, we show that the local time of $S^{H, K}$ has a jointly continuous version.

Theorem 2.3. Assume $H \in(0,1)$ and $K \in(0,1)$. On each (time-)interval $[a, b] \subset[0, \infty), S^{H, K}$ admits a local time which satisfies

$$
\int_{\mathbb{R}} L^{2}([a, b], x) d x<\infty \quad \text { a.s. }
$$

For the proof of Theorem 2.3 we need the following lemma. This result on the regularity of the increments of $S^{H, K}$ will be the key for the existence and the regularity of local times. 
Lemma 2.4. Assume $H \in(0,1)$ and $K \in(0,1)$. There exists $\delta>0$ and, for any integer $p \geq 2$, there exists a constant $0<C_{p}<+\infty$, such that

$$
\mathbb{E}\left[S_{t}^{H, K}-S_{s}^{H, K}\right]^{p} \geq C_{p}|t-s|^{p H K},
$$

for all $s, t \geq 0$ such that $|t-s|<\delta$.

Proof: By virtue of (3) and the elementary inequality $(a+b)^{2} \leq 2 a^{2}+2 b^{2}$, we have

$$
\mathbb{E}\left[S_{t}^{H K}-S_{s}^{H K}\right]^{2} \leq 2 \mathbb{E}\left[S_{t}^{H, K}-S_{s}^{H, K}\right]^{2}+2 C_{3}^{2}(K) \mathbb{E}\left[X_{t^{H}}^{K}-X_{s^{H}}^{K}\right]^{2} .
$$

Then, (2) implies that

$$
\mathbb{E}\left[S_{t}^{H, K}-S_{s}^{H, K}\right]^{2} \geq C_{H, K}|t-s|^{2 H K}-C_{3}^{2}(K) C_{K}\left|t^{H}-s^{H}\right|^{2} .
$$

For any $H \in(0,1)$, we have $\left|t^{H}-s^{H}\right| \leq|t-s|^{H}$, then

$$
\mathbb{E}\left[S_{t}^{H, K}-S_{s}^{H, K}\right]^{2} \geq|t-s|^{2 H K}\left[C_{H, K}-C_{3}^{2}(K) C_{K}|t-s|^{2 H(1-K)}\right] .
$$

Since $0<K<1$, we can choose $\delta>0$ small enough such that for all $t, s \geq 0$ with $|t-s|<\delta$, we have

$$
\left[C_{H, K}-C_{3}^{2}(K) C_{K}|t-s|^{2 H(1-K)}\right]>0 .
$$

Indeed, it suffices to choose

$$
\delta<\left(\frac{C_{H, K}}{C_{3}^{2}(K) C_{K}} \wedge 1\right)^{1 / 2 H(1-K)} .
$$

Finally,

$$
\mathbb{E}\left[S_{t}^{H, K}-S_{s}^{H, K}\right]^{2} \geq C|t-s|^{2 H K},
$$

with $|t-s|<\delta$ and

$$
C=\left[C_{H, K}-C_{3}^{2}(K) C_{K} \delta^{2 H(1-K)}\right] .
$$

Since $S^{H, K}$ is a centered Gaussian process, then the proof of Lemma 2.4.

Proof of Theorem 2.3: It is well known by Berman [2] that, for a jointly measurable zero-mean Gaussian process $X:=\{X(t) ; t \in[0,1]\}$ with bounded variance, the variance condition

$$
\int_{0}^{1} \int_{0}^{1}\left(\mathbb{E}[X(t)-X(t)]^{2}\right)^{-1 / 2} d s d t<\infty
$$


is sufficient for the local time $L(t, u)$ of $X$ to exist on $[0,1]$ a.s. and to be square integrable as a function of $u$. For any $[a, b] \subset[0, \infty)$, and for $I=\left[a^{\prime}, b^{\prime}\right] \subset[a, b]$ such that $\left|b^{\prime}-a^{\prime}\right|<\delta$, according to (5), we have,

$$
\int_{I} \int_{I}\left(\mathbb{E}\left[S^{H, K}(t)-S^{H, K}(s)\right]^{2}\right)^{-1 / 2} d s d t<C \int_{I} \int_{I}|t-s|^{-H K} d s d t .
$$

The last integral is finite because $0<H K<1$. Then $S^{H, K}$ possesses, on any interval $I \subset[a, b]$ with length $|I|<\delta$, a local time which is square integrable as function of $u$. Finally, since $[a, b]$ is a finite interval, we can obtain the local time on $[a, b]$ by a patch-up procedure, i.e. we partition $[a, b]$ into $\cup_{i=1}^{n}\left[a_{i-1}, a_{i}\right]$, such that $\left|a_{i}-a_{i-1}\right|<\delta$, and define $L([a, b], x)=\sum_{i=1}^{n} L\left(\left[a_{i-1}, a_{i}\right], x\right)$, where $a_{0}=a$ and $a_{n}=b$.

Proposition 2.5. Assume $H \in(0,1)$ and $K \in(0,1)$. Then $S^{H, K}$ is $L N D$ on $[0,1]$.

Proof: By virtue of (3), we have

$$
\left[S_{t}^{H K}-S_{s}^{H K}\right]=\left[S_{t}^{H, K}-S_{s}^{H, K}\right]+C_{3}(K)\left[X_{t^{H}}^{K}-X_{s^{H}}^{K}\right] .
$$

Therefore, the elementary inequality $(a+b)^{2} \leq 2 a^{2}+2 b^{2}$ implies that

$$
\begin{aligned}
\operatorname{Var}\left(\sum_{j=1}^{p} u_{j}\left[S_{t_{j}}^{H, K}-S_{t_{j-1}}^{H, K}\right]\right) \geq & \frac{1}{2} \operatorname{Var}\left(\sum_{j=1}^{p} u_{j}\left[S_{t_{j}}^{H K}-S_{t_{j-1}}^{H K}\right]\right) \\
& -C_{3}^{2}(K) \operatorname{Var}\left(\sum_{j=1}^{p} u_{j}\left[X_{t_{j}^{H}}^{K}-X_{t_{j-1}^{H}}^{K}\right]\right) .
\end{aligned}
$$

According to Remark 2.2, the $\operatorname{sfBm} S^{H K}$ is LND on $[0,1]$, then there exist two constants $\delta>0$ and $0<C_{p}<+\infty$ such that for any $t_{0}=0<$ $t_{1}<t_{2}<\cdots<t_{p}<1$ with $t_{p}-t_{1}<\delta$, we have

$$
\begin{aligned}
\operatorname{Var}\left(\sum_{j=1}^{p} u_{j}\left[S_{t_{j}}^{H, K}-S_{t_{j-1}}^{H, K}\right]\right) \geq & C_{p} \sum_{j=1}^{p} u_{j}^{2} \operatorname{Var}\left(S_{t_{j}}^{H K}-S_{t_{j-1}}^{H K}\right) \\
& -p C_{3}^{2}(K) \sum_{j=1}^{p} u_{j}^{2} \operatorname{Var}\left(X_{t_{j}^{H}}^{K}-X_{t_{j-1}^{H}}^{K}\right) .
\end{aligned}
$$


Moreover (2) and the fact that $H \in(0,1)$ imply that

$$
\begin{aligned}
\operatorname{Var}\left(\sum_{j=1}^{p}\right. & \left.u_{j}\left[S_{t_{j}}^{H, K}-S_{t_{j-1}}^{H, K}\right]\right) \\
& \geq C_{p} \sum_{j=1}^{p} u_{j}^{2}\left|t_{j}-t_{j-1}\right|^{2 H K}-p C_{3}^{2}(K) C_{K} \sum_{j=1}^{p} u_{j}^{2}\left|t_{j}-t_{j-1}\right|^{2 H} \\
& \geq\left[C_{p}-p C_{3}^{2}(K) C_{K} \delta^{2 H(1-K)}\right] \sum_{j=1}^{p} u_{j}^{2}\left|t_{j}-t_{j-1}\right|^{2 H K} .
\end{aligned}
$$

In addition, the elementary inequality $(a+b)^{2} \geq \frac{1}{2} a^{2}-b^{2}$ implies that

$$
\begin{aligned}
\mathbb{E}\left[S_{t}^{H, K}-S_{s}^{H, K}\right]^{2} & \leq 2 \mathbb{E}\left[S_{t}^{H K}-S_{s}^{H K}\right]^{2}+2 C_{3}^{2}(K) \mathbb{E}\left[X_{t^{H}}^{K}-X_{s^{H}}^{K}\right]^{2} \\
& \leq C_{H, K}|t-s|^{2 H K}+2 C_{3}^{2}(K) C_{K}\left|t^{H}-s^{H}\right|^{2} \\
& \leq\left[C_{H, K}+2 C_{3}^{2}(K) C_{K} \delta^{2 H(1-K)}\right]|t-s|^{2 H K} \\
& \leq C(H, K, \delta)|t-s|^{2 H K} .
\end{aligned}
$$

Therefore, it suffices now to choose

$$
\tilde{\delta}<\left(\frac{C_{p}}{p C_{3}^{2}(K) C_{K}}\right)^{\frac{1}{2 H(1-K)}} \wedge \delta
$$

and to consider

$$
C=\frac{1}{C(H, K, \delta)}\left[C_{p}-p C_{3}^{2}(K) C_{K} \tilde{\delta}^{2 H(1-K)}\right] .
$$

This with Proposition 2.1 complete the proof of Proposition 2.5.

Now, we are in position to give the main result of this section.

Theorem 2.6. Assume $H \in(0,1)$ and $K \in(0,1)$ and let $\delta>0$ the constant appearing in Lemma 2.4. For any integer $p \geq 2$ there exists a constant $C_{p}>0$ such that, for any $t \geq 0$, any $h \in(0, \delta)$, all $x, y \in \mathbb{R}$, and any $0<\xi<\frac{1-H K}{2 H K}$,

(6) $\mathbb{E}[L(t+h, x)-L(t, x)]^{p} \leq C_{p} \frac{h^{p(1-H K)}}{\Gamma(1+p(1-H K))}$,

(7) $\mathbb{E}[L(t+h, y)-L(t, y)-L(t+h, x)+L(t, x)]^{p}$

$$
\leq C_{p}|y-x|^{p \xi} \frac{h^{p(1-H K(1+\xi))}}{\Gamma(1+p(1-H K(1+\xi)))} .
$$


Proof: We will prove only (7), the proof of (6) is similar. It follows from (4) that for any $x, y \in \mathbb{R}, t, t+h \geq 0$ and for any integer $p \geq 2$,

$$
\begin{aligned}
\mathbb{E}[L(t+h, y)- & L(t, y)-L(t+h, x)+L(t, x)]^{p} \\
=\frac{1}{(2 \pi)^{p}} \int_{[t, t+h]^{p}} \int_{\mathbb{R}^{p}} \prod_{j=1}^{p}\left[e^{-i y u_{j}}-e^{-i x u_{j}}\right] & \\
& \times \mathbb{E}\left(e^{i \sum_{j=1}^{p} u_{j} S_{s_{j}}^{H, K}}\right) \prod_{j=1}^{p} d u_{j} \prod_{j=1}^{p} d s_{j} .
\end{aligned}
$$

Using the elementary inequality $\left|1-e^{i \theta}\right| \leq 2^{1-\xi}|\theta|^{\xi}$ for all $0<\xi<1$ and any $\theta \in \mathbb{R}$, we obtain

$$
\begin{aligned}
& \mathbb{E}[L(t+h, y)-L(t, y)-L(t+h, x)+L(t, x)]^{p} \leq\left(2^{\xi} \pi\right)^{-p} p !|x-y|^{p \xi} \\
& \quad \times \int_{t<t_{1}<\cdots<t_{p}<t+h} \int_{\mathbb{R}^{p}} \prod_{j=1}^{p}\left|u_{j}\right|^{\xi} \mathbb{E}\left[\exp \left(i \sum_{j=1}^{p} u_{j} S_{t_{j}}^{H, K}\right)\right] \prod_{j=1}^{p} d u_{j} \prod_{j=1}^{p} t_{j},
\end{aligned}
$$

where in order to apply the LND property of $S_{t}^{H, K}$, we replaced the integration over the domain $[t, t+h]$ by over the subset $t<t_{1}<\cdots<$ $t_{p}<t+h$. We deal now with the inner multiple integral over the $u$ 's. Change the variables of integration by mean of the transformation

$$
u_{j}=v_{j}-v_{j+1}, j=1, \ldots, p-1 ; u_{p}=v_{p} .
$$

Then, the linear combination in the exponent in (8) is transformed according to

$$
\sum_{j=1}^{p} u_{j} S_{t_{j}}^{H, K}=\sum_{j=1}^{p} v_{j}\left(S_{t_{j}}^{H, K}-S_{t_{j-1}}^{H, K}\right),
$$

where $t_{0}=0$. Since $S^{H, K}$ is a Gaussian process, the characteristic function in (8) has the form

$$
\exp \left(-\frac{1}{2} \operatorname{Var}\left[\sum_{j=1}^{p} v_{j}\left(S_{t_{j}}^{H, K}-S_{t_{j-1}}^{H, K}\right)\right]\right) .
$$

Since $|x-y|^{\xi} \leq|x|^{\xi}+|y|^{\xi}$ for all $0<\xi<1$, it follows that

$$
\prod_{j=1}^{p}\left|u_{j}\right|^{\xi} \leq \prod_{j=1}^{p-1}\left(\left|v_{j}\right|^{\xi}+\left|v_{j+1}\right|^{\xi}\right)\left|v_{p}\right|^{\xi} \text {. }
$$

Moreover, the last product is at most equal to a finite sum of $2^{p-1}$ terms of the form $\prod_{j=1}^{p}\left|x_{j}\right|^{\xi \varepsilon_{j}}$, where $\varepsilon_{j}=0,1$ or 2 and $\sum_{j=1}^{p} \varepsilon_{j}=p$. 
Let us write for simplicity $\sigma_{j}^{2}=\mathbb{E}\left(S_{t_{j}}^{H, K}-S_{t_{j-1}}^{H, K}\right)^{2}$. Combining the result of Proposition 2.5, (9) and (10), we get that the integral in (8) is dominated by the sum over all possible choices of $\left(\varepsilon_{1}, \ldots, \varepsilon_{m}\right) \in$ $\{0,1,2\}^{m}$ of the following terms

$$
\int_{t<t_{1}<\cdots<t_{p}<t+h} \int_{\mathbb{R}^{p}} \prod_{j=1}^{p}\left|v_{j}\right|^{\xi \varepsilon_{j}} \exp \left(-\frac{C_{p}}{2} \sum_{j=1}^{p} v_{j}^{2} \sigma_{j}^{2}\right) \prod_{j=1}^{p} d t_{j} d v_{j},
$$

where $C_{p}$ is the constant given in Proposition 2.5. The change of variable $x_{j}=\sigma_{j} v_{j}$ converts the last integral to

$$
\begin{aligned}
\int_{t<t_{1}<\cdots<t_{p}<t+h} \prod_{j=1}^{p} \sigma^{-1-\xi \varepsilon_{j}} d t_{1} \ldots d t_{p} & \\
& \times \int_{\mathbb{R}^{p}} \prod_{j=1}^{p}\left|x_{j}\right|^{\xi \varepsilon_{j}} \exp \left(-\frac{C_{p}}{2} \sum_{j=1}^{p} x_{j}^{2}\right) \prod_{j=1}^{p} d x_{j} .
\end{aligned}
$$

Let us denote

$$
J(p, \xi)=\int_{\mathbb{R}^{p}} \prod_{j=1}^{p}\left|x_{j}\right|^{\xi \varepsilon_{j}} \exp \left(-\frac{C_{p}}{2} \sum_{j=1}^{p} x_{j}^{2}\right) \prod_{j=1}^{p} d x_{j} .
$$

Consequently

$$
\begin{aligned}
& \mathbb{E}[L(t+h, y)-L(t, y)-L(t+h, x)+L(t, x)]^{p} \\
& \quad \leq J(p, \xi) C_{p}|y-x|^{p \xi} \int_{t<t_{1}<\cdots<t_{p}<t+h} \prod_{j=1}^{m} \sigma_{j}^{-1-\xi \varepsilon_{j}} d t_{1} \ldots d t_{p} .
\end{aligned}
$$

According to (5), for $h$ sufficiently small, namely $0<h<\inf (\delta, 1)$, we have

$$
\mathbb{E}\left[S_{t_{i}}^{H, K}-S_{t_{j}}^{H, K}\right]^{2} \geq C\left|t_{i}-t_{j}\right|^{2 H K}, \quad \forall t_{i}, t_{j} \in[t, t+h] .
$$

It follows that the integral on the right hand side of (11) is bounded, up to a constant, by

$$
\int_{t<t_{1}<\cdots<t_{p}<t+h} \prod_{j=1}^{p}\left(t_{j}-t_{j-1}\right)^{-H K\left(1+\xi \varepsilon_{j}\right)} d t_{1} \ldots d t_{p} .
$$

Since, $\left(t_{j}-t_{j-1}\right)<1$, for all $j \in\{2, \ldots, p\}$, we have

$$
\left(t_{j}-t_{j-1}\right)^{-H K\left(1+\xi \varepsilon_{j}\right)} \leq\left(t_{j}-t_{j-1}\right)^{-H K(1+2 \xi)}, \quad \forall \epsilon_{j} \in\{0,1,2\} .
$$


Since by hypothesis $0<\xi<\frac{1}{2 H K}-\frac{1}{2}$, the integral in (12) is finite. Moreover, by an elementary calculation, for all $p \geq 1, h>0$ and $b_{j}<1$,

$\int_{t<s_{1}<\cdots<s_{p}<t+h} \prod_{j=1}^{p}\left(s_{j}-s_{j-1}\right)^{-b_{j}} d s_{1} \ldots d s_{p}=h^{p-\sum_{j=1}^{p} b_{j}} \frac{\prod_{j=1}^{p} \Gamma\left(1-b_{j}\right)}{\Gamma\left(1+h-\sum_{j=1}^{p} b_{j}\right)}$

where $s_{0}=t$. It follows that (12) is dominated by

$$
C_{p} \frac{h^{p(1-H K(1+\xi))}}{\Gamma(1+p(1-H K(1+\xi))}
$$

where $\sum_{j=1}^{p} \varepsilon_{j}=p$. Consequently

$$
\begin{aligned}
\mathbb{E}[L(t+h, y)-L(t, y)-L( & +h, x)+L(t, x)]^{p} \\
& \leq C_{p}|y-x|^{p \xi} \frac{h^{p(1-H K(1+\xi))}}{\Gamma(1+p(1-H K(1+\xi)))} .
\end{aligned}
$$

Remark 2.7. Using the fact that $L(0, x)=0$ a.s. for any $x \in \mathbb{R}$ and $(7)$ by changing $t+h$ by $t$ and $t$ by 0 , we get

$$
\mathbb{E}[L(t, x)-L(t, y)]^{p} \leq C_{p} \frac{|x-y|^{p \xi}}{\Gamma(1+p(1-H K(1+\xi)))} .
$$

Acknowledgements. The author would like to thank the anonymous referee for her/his careful reading of the manuscript and useful comments.

\section{References}

[1] X. Bardina And D. Bascompte, A decomposition and weak approximation of the sub-fractional Brownian motion, Preprint, Departament de Matemàtiques, UAB (2009).

[2] S. M. Berman, Local times and sample function properties of stationary Gaussian processes, Trans. Amer. Math. Soc. 137 (1969), 277-299. DOI : 10.1090/S0002-9947-1969-0239652-5.

[3] S. M. Berman, Local nondeterminism and local times of Gaussian processes, Indiana Univ. Math. J. 23 (1973/74), 69-94.

[4] T. Bojdecki, L. G. Gorostiza, and A. Talarczyk, Subfractional Brownian motion and its relation to occupation times, Statist. Probab. Lett. 69(4) (2004), 405-419. DOI: 10.1016/j.spl. 2004.06.035.

[5] D. Geman and J. Horowitz, Occupation densities, Ann. Probab. 8(1) (1980), 1-67. 
[6] C. Houdré And J. Villa, An example of infinite dimensional quasi-helix, in: "Stochastic models" (Mexico City, 2002), Contemp. Math. 336, Amer. Math. Soc., Providence, RI, 2003, pp. 195-201. DOI : $10.1090 /$ conm/336/06034.

[7] I. Mendy, On the local time of sub-fractional Brownian motion, Ann. Math. Blaise Pascal 17(2) (2010), 357-374. DoI: 10.5802/ ambp. 288.

[8] J. Ruiz de Chávez And C. Tudor, A decomposition of subfractional Brownian motion, Math. Rep. (Bucur.) 11(61), no. 1 (2009), 67-74.

Faculté des Sciences Oujda

Laboratoire de Modélisation Stochastique et Déterministe

et URAC 04

B.P. 717

Maroc

E-mail address: semastai@hotmail.fr

Primera versió rebuda el 9 de juliol de 2012, darrera versió rebuda el 17 de setembre de 2012 . 